\title{
Utility of Beamforming Strategies for Secrecy in Multiuser MIMO Wiretap Channels
}

\author{
Amitav Mukherjee and A. Lee Swindlehurst \\ Dept. of Electrical Engineering \& Computer Science \\ University of California \\ Irvine, CA 92697-2625
}

\begin{abstract}
This paper examines linear beamforming methods for secure communications in a multiuser wiretap channel with a single transmitter, multiple legitimate receivers, and a single eavesdropper, where all nodes are equipped with multiple antennas. No information regarding the eavesdropper is presumed at the transmitter, and we examine both the broadcast MIMO downlink with independent information, and the multicast MIMO downlink with common information for all legitimate receivers. In both cases the information signal is transmitted with just enough power to guarantee a certain SINR at the desired receivers, while the remainder of the power is used to broadcast artificial noise. The artificial interference selectively degrades the passive eavesdropper's signal while remaining orthogonal to the desired receivers. We analyze the confidentiality provided by zero-forcing and optimal minimum-power beamforming designs for the broadcast channel, and optimal minimum-MSE beamformers for the multicast channel. Numerical simulations for the relative SINR and BER performance of the eavesdropper demonstrate the effectiveness of the proposed physicallayer security schemes.
\end{abstract}

\section{INTRODUCTION}

The growing interest in security at the physical layer of wireless communications has sparked a resurgence of research in information-theoretic secrecy. Physical layer security incorporates signal and code design to limit the information that can be extracted by an eavesdropper at the bit level, as a supplement to classical cryptographic security at the link or higher layers. Wyner's landmark paper on secure communications in a point-to-point wiretap channel [1] paved the way for characterizing the secrecy capacity of specific types of multiuser broadcast, interference, and multiple-access channels with singleantenna nodes [2]-[6], although their general secrecy capacity regions under fading remain mostly unknown. Similarly, the secrecy capacity achievable in multipleinput multiple-output (MIMO) multiuser networks is largely an open problem, with limited results available for MIMO broadcast channels with two receivers
[7]. In the majority of the literature on confidential transmissions in multiuser networks, knowledge of the probability distribution of the eavesdropper's channel is assumed at the transmitter, which inherently provides information about the number of eavesdropper antennas as well.

Motivated by the above, this paper studies the effectiveness of simple beamforming strategies for maintaining confidentiality in a MIMO downlink system with multiple legitimate multi-antenna receivers and a single passive eavesdropper with an unknown channel distribution. A portion of the transmit power is used to broadcast the information signal vector with just enough power to guarantee a certain signal-to-interference-plus-noise ratio (SINR) for the intended receivers, and the remainder of the power is used to broadcast artificial noise in order to mask the desired signal from a potential eavesdropper. The artificial interference is designed to be orthogonal to the information-bearing signals at the intended receivers, which ensures that only the eavesdropper suffers a SINR penalty. Jamming potential eavesdroppers with artificial noise has been previously proposed for a point-to-point MIMO wiretap channel in [8], [9].

For the MIMO broadcast channel with independent signals, we compare the power efficiency and relative SINR of two different approaches: a zero-forcing beamforming design, and an iterative minimum-power joint transmit-receive beamformer design with a minimum SINR constraint per user. The zero-forcing solution allocates slightly lower power for artificial noise at low transmit power levels, but enjoys a significant advantage in terms of complexity. For the MIMO multicast channel, an iterative minimum-power optimal beamformer design is employed with a minimum mean square error (MMSE) criterion for each user.

In the next section, the mathematical models for the MIMO broadcast and multicast channels are presented. The known algorithms for zero-forcing beamforming and the optimal minimum-power beamformer design are outlined in Section III The wiretapping strategies that 
a potential eavesdropper could employ are described in Section IV. The resulting system performance is studied via simulation in Section $\mathrm{V}$, and concluding remarks are presented in Section VI

Notation: $\mathcal{E}\{\cdot\}$ denotes expectation, $(\cdot)^{T}$ the transpose, $(\cdot)^{H}$ the Hermitian transpose, $\operatorname{Tr}(\cdot)$ is the trace operator, $[\mathbf{A}]_{p, q}$ denotes the $(p, q)$ entry of matrix $\mathbf{A}$, $\operatorname{diag}\{\mathbf{x}\}$ is a diagonal matrix with vector $\mathbf{x}$ on the diagonal, $\|\cdot\|_{2}$ is the Euclidean norm, $\mathbf{1}$ is a column vector of ones, and I is an identity matrix of appropriate dimension.

\section{MATHEMATICAL MODEL}

The network under consideration is comprised of a $N_{t}$-antenna transmitter broadcasting to $K$ legitimate receivers with $N_{r}$ antennas each, and a single passive eavesdropper with $N_{e}$ antennas in the vicinity of the network. For the moment, we assume $K<N_{t}$, and also remark that the proposed secrecy scheme is valid for an arbitrary number of eavesdroppers. The transmitter is assumed to have perfect channel state information (CSI) for all of the intended receivers, but is unaware of either the instantaneous CSI or the distribution of the CSI for the eavesdropper. This lack of information precludes the use of secrecy capacity as our performance metric, thus we choose to work directly with SINR. The transmitter's primary objective is to allow each of the desired receivers to recover the transmitted data with a certain SINR, while denying the eavesdropper as much information as possible about the data. Similar to the approaches taken in [8], [9], this will be accomplished by transmission of a jamming signal simultaneously with the data intended for the desired receivers.

We will treat two separate cases: (1) transmission of a unique data symbol to each of the $K$ users, referred to as 'broadcasting', and (2) sending the same common information signal to all receivers, referred to as 'multicasting.' The data model for these two scenarios is detailed below.

\section{A. MIMO Broadcast Channel}

In the broadcast scenario considered here, the transmitter wishes to send a private, independent scalar message $z_{k}$ to each receiver. The transmitter employs a linear $N_{t} \times 1$ transmit beamformer $\mathbf{t}_{k}$ for the information symbol $z_{k}$ of each intended receiver, and is assumed to have a total power constraint $P$ encompassing information transmission and jamming. We denote the information signal by the $K \times 1$ vector $\mathbf{z}=\left(z_{1}, \ldots, z_{K}\right)^{T}$, and the jamming signal by the $N_{t} \times 1$ vector $\mathbf{z}^{\prime}$. Assume unit-norm transmit beamformers $\mathbf{t}_{k}^{H} \mathbf{t}_{k}=1$, and symbol power $\mathcal{E}\left\{\left|z_{k}\right|^{2}\right\}=\rho_{k} P$, where $0<\rho_{k} \leq 1$ is the fraction of the power devoted to the information signal of user $k$, and $\rho \equiv \sum_{k=1}^{K} \rho_{k}$ is the fraction of the total power used for information transmission.

Define

$$
\mathcal{E}\left\{\mathbf{z}^{\prime} \mathbf{z}^{\prime H}\right\}=\mathbf{Q}_{z}^{\prime} \quad \operatorname{Tr}\left(\mathbf{Q}_{z}^{\prime}\right)=(1-\rho) P,
$$

and let $\mathbf{T}=\left[\begin{array}{lll}\mathbf{t}_{1} & \ldots & \mathbf{t}_{K}\end{array}\right]$ denote the aggregate transmit beamforming matrix. The signal broadcast by the transmitter is then given by

$$
\mathbf{x}=\mathbf{T z}+\mathbf{z}^{\prime} .
$$

In a flat-fading scenario, the received signal at the $k^{t h}$ legitimate receiver, $k=1, \ldots, K$, can be written as

$$
\mathbf{y}_{k}=\mathbf{H}_{k} \mathbf{t}_{k} z_{k}+\sum_{j \neq k}^{K} \mathbf{H}_{k} \mathbf{t}_{j} z_{j}+\mathbf{H}_{k} \mathbf{z}^{\prime}+\mathbf{n}_{k},
$$

where $\mathbf{H}_{k}$ is the corresponding $N_{r} \times N_{t}$ channel matrix between the transmitter and user $k$, and $\mathbf{n}_{k}$ is the naturally occurring i.i.d additive white Gaussian noise vector with covariance $\mathcal{E}\left\{\mathbf{n}_{b} \mathbf{n}_{b}^{H}\right\}=\sigma_{n}^{2} \mathbf{I}$. Analogous parameters can be defined for the eavesdropper, who receives

$$
\mathbf{y}_{e}=\mathbf{H}_{e} \sum_{j=1}^{K} \mathbf{t}_{j} z_{j}+\mathbf{H}_{e} \mathbf{z}^{\prime}+\mathbf{n}_{e} .
$$

The $k^{\text {th }}$ receiver uses a $N_{r} \times 1$ beamformer $\mathbf{w}_{k}$ to recover its information, which leads to the decision variable

$$
\hat{z}_{k}=\mathbf{w}_{k}^{H} \mathbf{H}_{k} \mathbf{t}_{k} z_{k}+\mathbf{w}_{k}^{H} \mathbf{H}_{k} \sum_{j \neq k}^{K} \mathbf{t}_{j} z_{j}+\mathbf{w}_{k}^{H} \mathbf{H}_{k} \mathbf{z}^{\prime}+\mathbf{w}_{k}^{H} \mathbf{n}_{k} .
$$

\section{B. MIMO Multicast Channel}

In the case of multicast, a common information symbol $z$ with power $\mathcal{E}\left\{|z|^{2}\right\}=\rho P$ is transmitted to all $K$ receivers. This necessitates the use of a common $N_{t} \times 1$ transmit beamformer $\mathbf{u}$, with $\mathbf{u}^{H} \mathbf{u}=1$. Assuming the same power constraints and artificial noise properties as in Section $\amalg-\mathrm{A}$, the transmitted signal is

$$
\mathbf{x}=\mathbf{u} z+\mathbf{z}^{\prime} .
$$

The received signals are now

$$
\begin{aligned}
& \mathbf{y}_{k}=\mathbf{H}_{k} \mathbf{u} z+\mathbf{H}_{k} \mathbf{z}^{\prime}+\mathbf{n}_{k} \\
& \mathbf{y}_{e}=\mathbf{H}_{e} \mathbf{u} z+\mathbf{H}_{e} \mathbf{z}^{\prime}+\mathbf{n}_{e},
\end{aligned}
$$

and the $k^{t h}$ receiver employs a $N_{r} \times 1$ beamformer $\mathbf{r}_{k}$ to obtain its decision variable as

$$
\hat{z}_{k}=\mathbf{r}_{k}^{H} \mathbf{H}_{k} \mathbf{u} z+\mathbf{r}_{k}^{H} \mathbf{H}_{k} \mathbf{z}^{\prime}+\mathbf{r}_{k}^{H} \mathbf{n}_{k} .
$$




\section{Artificial Noise}

As will be discussed in the next section, our goal will be to minimize the total transmit power required to achieve a certain SINR for each receiver, and use all remaining power to jam the eavesdropper. To achieve this goal, we will choose the transmit and receive beamformers in such a way that the jamming signal does not impact the quality of the desired receiver's signal. Assuming the transmitter is aware of the beamformers used by each of the receivers, an effective downlink channel to the $K$ receivers can be constructed for either the broadcast or multicast case as follows:

$$
\tilde{\mathbf{H}}=\left[\begin{array}{lll}
\tilde{\mathbf{H}}_{1} & \ldots & \tilde{\mathbf{H}}_{K}
\end{array}\right]^{T}
$$

where $\tilde{\mathbf{H}}_{k}=\left(\mathbf{w}_{k}^{H} \mathbf{H}_{k}\right)^{T}$ or $\tilde{\mathbf{H}}_{k}=\left(\mathbf{r}_{k}^{H} \mathbf{H}_{k}\right)^{T}$. When $K<N_{t}$, the jamming signal $\mathbf{z}^{\prime}$ can be chosen from the nullspace of $\tilde{\mathbf{H}}$ in order to guarantee that it does not impact the desired receivers. If $K \geq N_{t}$, the nullspace of the effective downlink channel does not exist in general, and the artificial noise could not be guaranteed to be orthogonal to the desired signals. Although the artificial noise can still be constructed so as to minimize its impact at the receivers (e.g., by forcing it to lie in the right subspace of $\tilde{\mathbf{H}}$ with smallest singular value), a scheduling strategy to ensure $K<N_{t}$ may be more appropriate in the context of this work. We note that user scheduling in wireless networks with secrecy considerations has received limited attention thus far.

\section{BEAMFORMING DESIGN}

In either the broadcast or multicast case, the design of the transmit and receive beamformers is, in general, coupled; that is, the choice of $\mathbf{t}_{k}, \mathbf{u}$ depends on the choice of $\mathbf{w}_{k}, \mathbf{r}_{k}$ respectively, and vice versa. One solution to this problem is to fix the beamformer on one end of the link and then optimize the other. An optimal approach would design the beamformers jointly, although at the expense of increased complexity [12][15]. In this paper, we consider both types of approaches. In the first, zero-forcing at the transmitter is used for design of the transmit beamformers in the broadcast case; this eliminates multi-user interference at each receiver, and leads to a simple maximum-ratio combiner at each receiver. In the second approach, we consider the optimal joint beamformer design problem for both the broadcast and multicast cases. The lack of eavesdropper CSI preempts the development of beamforming designs tailored towards maximizing a particular secrecy metric; hence we utilize existing precoding methods as discussed in the sequel.

\section{A. Zero-Forcing Beamforming}

In this section, we adopt a modified version of the coordinated zero-forcing beamforming approach in [11]. Assume that $K<N_{t}$, and for user $k$, define $\tilde{\mathbf{H}}_{k}$ as

$$
\begin{aligned}
& \tilde{\mathbf{H}}_{k}=\left[\begin{array}{llllll}
\tilde{\mathbf{h}}_{1} & \ldots & \tilde{\mathbf{h}}_{k-1} & \tilde{\mathbf{h}}_{k+1} & \ldots & \tilde{\mathbf{h}}_{K}
\end{array}\right] \\
& \tilde{\mathbf{h}}_{l}=\left(\mathbf{w}_{l}^{H} \mathbf{H}_{l}\right)^{T}
\end{aligned}
$$

The singular value decomposition (SVD) of $\tilde{\mathbf{H}}_{k}$ yields

$$
\tilde{\mathbf{H}}_{k}=\tilde{\mathbf{U}}_{k} \tilde{\mathbf{D}}_{k}\left[\begin{array}{ll}
\tilde{\mathbf{V}}_{k}^{(s)} & \tilde{\mathbf{v}}_{k}^{(0)}
\end{array}\right]^{H},
$$

where $\tilde{\mathbf{U}}_{k}$ is the matrix of left singular vectors, $\tilde{\mathbf{D}}_{k}$ is the diagonal matrix of singular values, $\tilde{\mathbf{v}}_{k}^{(0)}$ is the right singular vector associated with the smallest (zero) singular value, and $\tilde{\mathbf{V}}_{k}^{(s)}$ is the collection of right singular vectors corresponding to other singular values.

Evidently, $\mathbf{v}_{k}^{(0)}$ is a logical choice for the $k^{t h}$ transmit beamformer $\mathbf{t}_{k}$ given the objective of nulling all multiuser interference. Since receiver $k$ then sees only its desired signal in spatially white noise, the optimal receive beamformer $\mathbf{w}_{k}$ is simply the maximum-ratio combiner:

$$
\mathbf{w}_{k}=\mathbf{H}_{k} \mathbf{t}_{k}
$$

Assuming that the jamming signal is orthogonal to $\mathbf{w}_{k}^{H} \mathbf{H}_{k}$, the SINR at user $k$ can then be written as

$$
\operatorname{SINR}_{k}=\frac{\rho_{k} P\left|\mathbf{w}_{k}^{H} \mathbf{H}_{k} \mathbf{t}_{k}\right|^{2}}{\sigma_{n}^{2} \mathbf{w}_{k}^{H} \mathbf{w}_{k}} .
$$

For a target SINR $S_{k}$, the required power allocation for user $k$ can be calculated as

$$
\rho_{k}=\frac{\sigma_{n}^{2} S_{k}}{\mathbf{t}_{k}^{H} \mathbf{H}_{k}^{H} \mathbf{H}_{k} \mathbf{t}_{k} P} .
$$

\section{B. Optimal Minimum Transmit Power Beamforming with per-user SINR constraint}

Although relatively simple, the proposed zero-forcing algorithm in Section ஹI-A will not in general minimize $\rho P$. To minimize the transmit power necessary to achieve the desired SINR, it is necessary to jointly design the transmit and receive beamformers [12]-[15]. Since the optimal beamformers will not be of the zeroforcing type, the downlink beamformer design problem is non-convex due to the interdependence of the problem variables. This issue can be overcome by exploiting the SINR duality of the downlink and uplink channels, which states that the minimum sum power required to achieve a set of SINR values on the downlink is equal to the minimum sum power required to achieve the same SINR vector on the dual uplink channel [15]. Therefore, as 
a benchmark we compute the optimum transmit/receive beamformers and power allocation that minimizes the sum transmit power while satisfying the SINR constraint per user based on [14], [15].

Let $\mathbf{t}_{k}^{(i)}, \mathbf{w}_{k}^{(i)}, p_{k}^{(i)}$ and $q_{k}^{(i)}$ represent the transmit/receive beamformers and downlink/uplink power allocation for user $k$ at the $i^{\text {th }}$ iteration. Define $g_{s, k}$ for $s, k=1, \ldots, K$ as the signal and interference powers for each user:

$$
g_{s, k} \triangleq\left\{\begin{array}{c}
\left|\left(\mathbf{t}_{s}^{(i)}\right)^{H} \mathbf{H}_{k, s}^{H} \mathbf{w}_{k}^{(i)}\right|^{2} \text { for uplink } \\
\left|\left(\mathbf{w}_{k}^{(i)}\right)^{H} \mathbf{H}_{k, s} \mathbf{t}_{s}^{(i+1)}\right|^{2} \text { for downlink }
\end{array}\right.
$$

The SINR per stream on either link can then be written as

$$
\gamma_{k}=\frac{x_{k}\left|g_{s, s}\right|^{2}}{1+\sum_{i=1, i \neq s}^{K} x_{i}\left|g_{s, i}\right|^{2}}
$$

where $x_{k}$ is the power allotted to user $k$ either on the downlink or the dual uplink channel. Finally, define matrices $\mathbf{C}$ and $\mathbf{D}$ as

$$
\begin{gathered}
{[\mathbf{C}]_{s, k}=\left\{\begin{array}{cc}
g_{s, k} & \text { if } s \neq k \\
0 & \text { if } s=k
\end{array}\right.} \\
\mathbf{D}=\operatorname{diag}\left\{\frac{\gamma_{t h}}{g_{1,1}}, \ldots, \frac{\gamma_{t h}}{g_{K, K}}\right\} .
\end{gathered}
$$

The iterative beamformer design that minimizes the transmit power can be summarized as follows.

1) Set the initial transmit beamformers to the zeroforcing solution $\mathbf{t}_{k}^{(0)}=\tilde{\mathbf{v}}_{k}^{(0)}$, with an initial power allocation $p_{k}^{(0)}=P / K$ per user. Compute the optimum set of receive beamformers as

$$
\mathbf{w}_{k}^{(i)}=\sqrt{p_{k}^{(i)}} \mathbf{t}_{k}^{(i) H} \mathbf{H}_{k}^{H}\left(\sum_{i=1}^{K} p_{k}^{(i)} \mathbf{H}_{k} \mathbf{t}_{k}^{(i)} \mathbf{t}_{k}^{(i) H} \mathbf{H}_{k}^{H}+\mathbf{I}\right)
$$

2) Now consider the dual uplink channel where the transmit beamformers from Step 1 serve as the receive beamformers, and vice versa. Update the power allocation vector $\mathbf{q}^{(i+1)}=\mathbf{x}^{*}$ from

$$
\mathbf{x}^{*}=(\mathbf{I}-\mathbf{D C})^{-1} \mathbf{D} \mathbf{1},
$$

then update the beamformer set using (21):

$\mathbf{t}_{k}^{(i)}=\sqrt{q_{k}^{(i)}} \mathbf{w}_{k}^{(i) H} \mathbf{H}_{k}\left(\sum_{i=1}^{K} q_{k}^{(i)} \mathbf{H}_{k}^{H} \mathbf{w}_{k}^{(i)} \mathbf{w}_{k}^{(i) H} \mathbf{H}_{k}+\mathbf{I}\right)^{\mathbf{j}}$

Calculate the achieved SINR set on the uplink using (16), and go to Step 3.

3) Revert back to the downlink channel and recompute the power allocation vector $\mathbf{p}^{(i+1)}=\mathbf{x}^{*}$ using
(20). Calculate the downlink SINR set using (16) and compare with the uplink SINR vector from Step 2 for convergence. If the stopping criterion is not satisfied, set $i=i+1$ and return to Step 2, otherwise terminate the algorithm.

\section{Optimal Minimum Transmit Power Beamforming with per-user MSE constraint}

Despite superficial similarities, the beamforming design problems with per-user SINR constraints for the broadcast and multicast channels are fundamentally different. Many efficient numerical solutions exist for the former as cited in Section [II-A whereas the multicast problem is known to be non-convex even for the MISO case with single antennas at each receiver. A number of approximate solutions based on semidefinite relaxation techniques have been proposed for the MISO multicast channel, e.g. [16], [17].

However, the MIMO multicast beamforming problem was recently reformulated as a convex optimization by replacing the per-user SINR requirements with minimum MSE constraints [18]. In this case, the optimal receiver structure is known to be MMSE, which allows an alternating iterative optimization of the transmit and receive beamformers. The minimum sum-power optimization problem can be expressed as a convex second-order cone program (SOCP):

$$
\begin{aligned}
& \min _{\mathbf{u}, t} t \\
& \text { s.t. }\|\mathbf{u}\|_{2} \leqslant t, \\
& \left\|v_{k}\right\|_{2} \leqslant \sqrt{\varepsilon_{k}-w_{k}}, \forall k
\end{aligned}
$$

where $\varepsilon_{k}$ is the MMSE constraint per user, $v_{k}=$ $\mathbf{r}_{k}^{H} \mathbf{H}_{k} \mathbf{u}-1$, and $w_{k}=\sigma_{n}^{2} \operatorname{Tr}\left(\mathbf{r}_{k} \mathbf{r}_{k}^{H}\right)$.

In brief, the algorithm is initialized with random receive beamformers, after which the corresponding optimal transmit beamformer is computed by solving (22). The receive beamformers are updated using the MMSE criterion as

$$
\mathbf{r}_{k}=\mathbf{u}^{H} \mathbf{H}_{k}^{H}\left(\mathbf{H}_{k} \mathbf{u} \mathbf{u}^{H} \mathbf{H}_{k}^{H}+\sigma_{n}^{2} \mathbf{I}\right)^{-1},
$$

and the iterations continue until convergence. For consistency with our choice of SINR as the performance metric, the following equivalence relation between maximum SINR and MMSE is useful:

$$
\varepsilon_{k}=\frac{1}{1+\operatorname{SINR}_{k}} .
$$

Remark 1: For both zero-forcing and joint minimumpower beamformer designs, the computations are carried out at the transmitter, which then needs to supply the receivers with information about the optimal beamformers. 
This can be done using a limited (quantized) feedforward scheme, as proposed in [19].

Remark 2: The assumption of perfect CSIT of the intended receivers is admittedly a strong one. Robust beamforming design for the MIMO downlink with multiantenna receivers is an ongoing research problem, with some recent results provided in [20]. However, incorporating artificial noise into any such robust beamforming schemes is not a straightforward extension, since the lack of exact receiver CSI at the transmitter would no longer allow any artificial noise to be perfectly orthogonal to the intended receivers. The authors have conducted a perturbation analysis to capture the performance degradation in single-user MIMO wiretap channels [21], and an extension to the downlink case is in progress.

Remark 3: As mentioned previously, the assumption that the number of receivers is less than the number of transmit antennas in Section II-C can be relaxed by implementing a user selection stage prior to transmission. The authors have shown in [22] that a greedy algorithm which schedules the user set based on maximizing the transmit power available for artificial noise performs close to an optimal exhaustive search in terms of eavesdropper BER.

\section{WIRETAPPING STRATEGIES}

We consider two types of eavesdropper strategies: (1) a simple linear receiver approach in which the eavesdropper attempts to maximize the SINR of the data stream she is interested in decoding, and (2) a multi-user decoding scheme in which the eavesdropper uses maximum likelihood detection to decode all $K$ information-bearing waveforms. We begin by illustrating approach (1) for the broadcast case, with the extension to the multicast channel being straightforward. Assume that the eavesdropper seeks to recover the data stream of user $k$ from her received signal given in (3). The interference-plus-noise covariance matrix given that $z_{k}$ is the symbol of interest is

$$
\mathbf{Q}_{e}^{k}=\mathbf{H}_{e} \sum_{j \neq k}^{K} \mathbf{t}_{j} \mathbf{t}_{j}^{H} \mathbf{H}_{e}^{H}+\mathbf{H}_{e} \mathbf{Q}_{z}^{\prime} \mathbf{H}_{e}^{H}+\sigma_{e}^{2} \mathbf{I} .
$$

The maximum SINR beamformer for the data stream of user $k$ is then given by

$$
\mathbf{w}_{e}=\left(\mathbf{Q}_{e}^{k}\right)^{-1} \mathbf{H}_{e} \mathbf{t}_{k} .
$$

The use of an optimal beamformer here presumes that $\mathbf{H}_{e} \mathbf{t}_{k}, k=1, \ldots, K$ is somehow known at the eavesdropper. Using this approach, the SINR at the eavesdropper can be calculated to be

$$
\operatorname{SINR}_{e, k}=\mathbf{t}_{k}^{H} \mathbf{H}_{e}^{H}\left(\mathbf{Q}_{e}^{k}\right)^{-1} \mathbf{H}_{e} \mathbf{t}_{k} .
$$

An eavesdropper with more extensive computational resources will attack the MIMO broadcast network using a more sophisticated approach. In general, the optimal decoder at the eavesdropper in terms of minimizing the symbol error rate would be the following Maximum Likelihood (ML) detector:

$$
\hat{\mathbf{z}}=\arg \min _{\mathbf{z} \in \mathcal{Z}}\left\{\left\|\mathbf{Q}_{e}^{-1 / 2}\left(\mathbf{y}_{e}-\mathbf{H}_{e} \mathbf{T} \mathbf{z}\right)\right\|^{2}\right\}
$$

where $\mathcal{Z}$ is the signal space from which $\mathbf{z}$ is drawn, and $\mathbf{Q}_{e}=\mathbf{H}_{e} \mathbf{Q}_{z}^{\prime} \mathbf{H}_{e}^{H}+\sigma_{e}^{2} \mathbf{I}$ is the interference-plus-noise covariance matrix perceived by the eavesdropper.

In the next section, we present numerical examples that show the SINR and the BER that the eavesdropper experiences with the proposed jamming scheme.

\section{SIMULATION RESULTS}

We investigate the performance of the eavesdropper and the desired receivers as a function of the target SINR at the receivers and the total available transmit power. In each simulation, we assume a transmitter with $N_{t}=4$ antennas, $K=3$ legitimate receivers with $N_{r}=2$ antennas each, and an eavesdropper with $N_{e}=4$ antennas. The channel matrices for all links are composed of independent Gaussian random variables with zero mean and unit variance. The background noise power is assumed to be the same for all $K$ receivers and the eavesdropper: $\sigma_{n}^{2}=\sigma_{e}^{2}=1$. The algorithm of [14] is used to implement the optimal joint beamformer design, and the SOCP in (22) was solved using the MATLAB cvx optimization toolbox [23]. All of the displayed results are calculated based on an average of 5000 trials with independent channel and noise realizations.

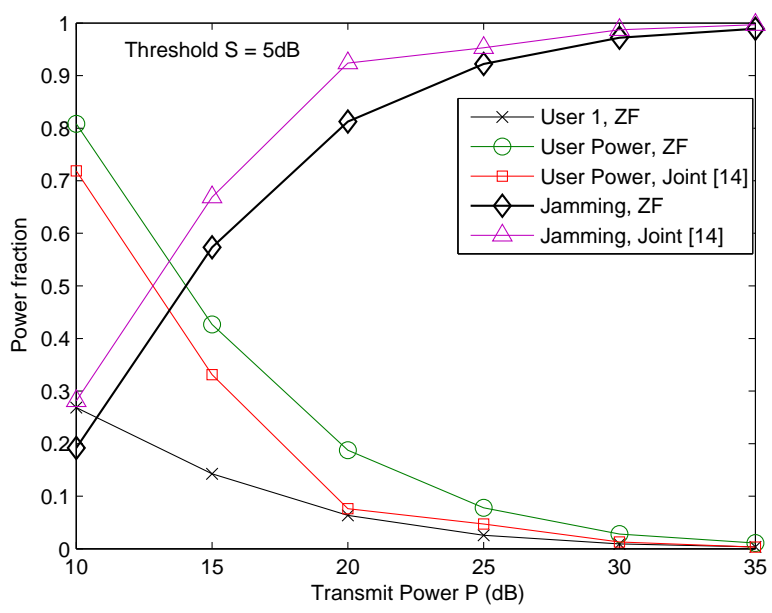

Fig. 1. Power fraction versus transmit power $P$ for $K=3$ users, $N_{t}=4, N_{r}=2, N_{e}=4$ antennas. 
Figure 1 displays the average fraction of the transmit power allocated to data and artificial noise by the zeroforcing and the optimal minimum transmit power beamforming algorithms with an SINR threshold of $5 \mathrm{~dB}$. The joint design requires roughly $10 \%$ less transmit power at small to intermediate power levels, albeit with a significantly greater level of complexity. The curve labeled "User 1,ZF" represents the fraction of the total power assigned to an arbitrary user (referred to as user 1) among the three legitimate receivers.

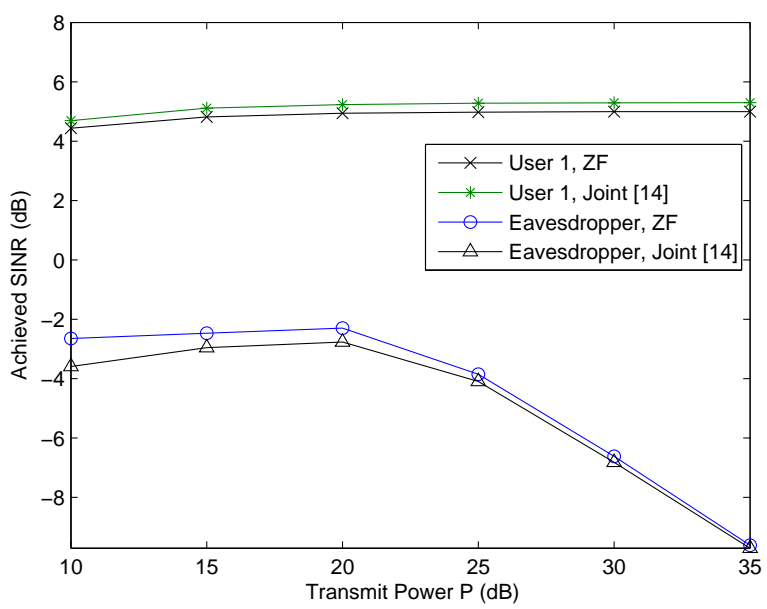

Fig. 2. SINR versus transmit power $P$ for $K=3$ users, $N_{t}=$ $4, N_{r}=2, N_{e}=4$ antennas.

Figure 2 shows the achieved SINR levels versus transmit power $P$ for user 1 and the average SINR averaged over all three streams for the eavesdropper when using single-user detection. The legitimate receiver almost always achieves the desired SINR target of $5 \mathrm{~dB}$, while the eavesdropper has a significantly lower SINR due to the artificial noise. The SINR of user 1 is slightly below $5 \mathrm{~dB}$ for transmit powers of 10 and $15 \mathrm{~dB}$, since there were a few trials for which the $5 \mathrm{~dB}$ SINR target could not be achieved. In such cases, the transmitter devotes all power to the desired receivers and none to jamming, and the resulting SINR is averaged in with the other trials. Note that there is not a significant difference in performance for the eavesdropper whether the zeroforcing or optimal broadcast beamformers are used.

Figure 3 compares the eavesdropper's BER with and without artificial noise when the eavesdropper employs MIMO ML detection, assuming an uncoded BPSKmodulated information signal $\mathbf{z}$ and zero-forcing transmit beamforming. For low target SINRs, we observe a significant degradation in the eavesdropper's interception capabilities, e.g., by approximately $10.5 \mathrm{~dB}$ at $B E R=0.05$. A more modest gain of $2.5 \mathrm{~dB}$ is achieved at $B E R=10^{-2}$ for intermediate target SINRs. At

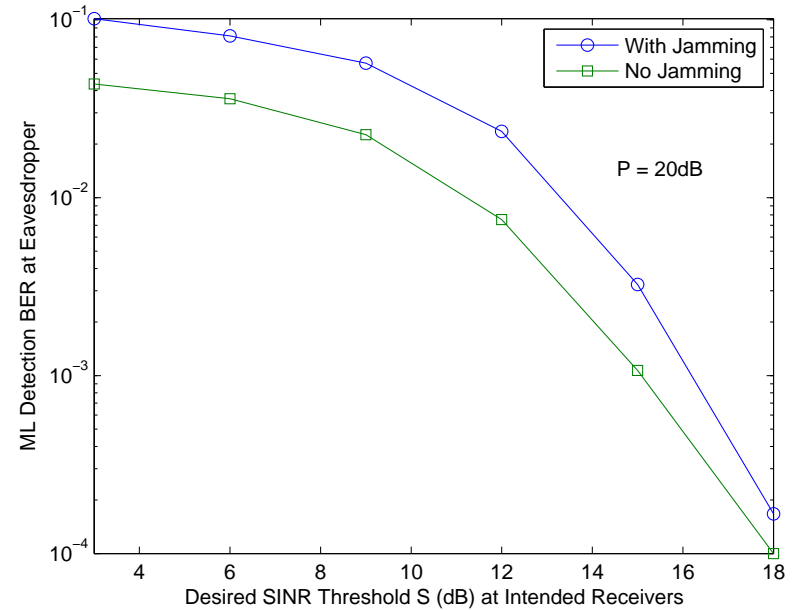

Fig. 3. Eavesdropper maximum-likelihood BER versus target SINR $S$ for $K=3$ users, $N_{t}=4, N_{r}=2, N_{e}=4$ antennas.

high SINR thresholds, the two curves converge since the transmitter is constrained to allocate progressively smaller fractions of the total power to jamming.

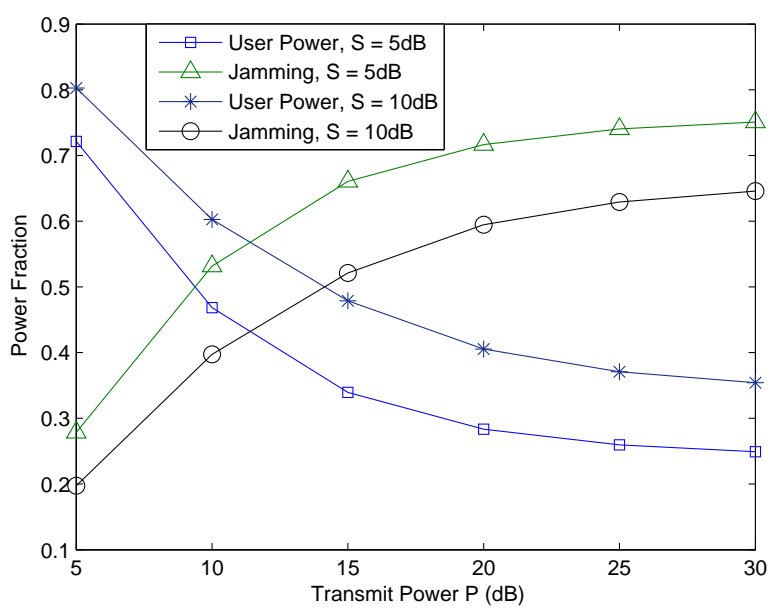

Fig. 4. Power fraction versus transmit power $P$ for $K=3$ users, $N_{t}=4, N_{r}=2, N_{e}=4$ antennas.

Figure 4 displays the fraction of the transmit power allocated to data and artificial noise by the multicast minimum transmit-power beamforming algorithm of Section III-C, with SINR thresholds of $S=5 \mathrm{~dB}, 10 \mathrm{~dB}$. The lack of inter-user interference allows the transmitter to allocate more power for jamming compared to the broadcast case for the same transmit power.

Figure 5 shows the average achieved SINR levels at the intended receivers and the eavesdropper versus transmit power $P$ for user SINR thresholds of $S=5$ $\mathrm{dB}, 10 \mathrm{~dB}$. As before, the legitimate receivers achieve the desired SINR targets, while the eavesdropper's per- 


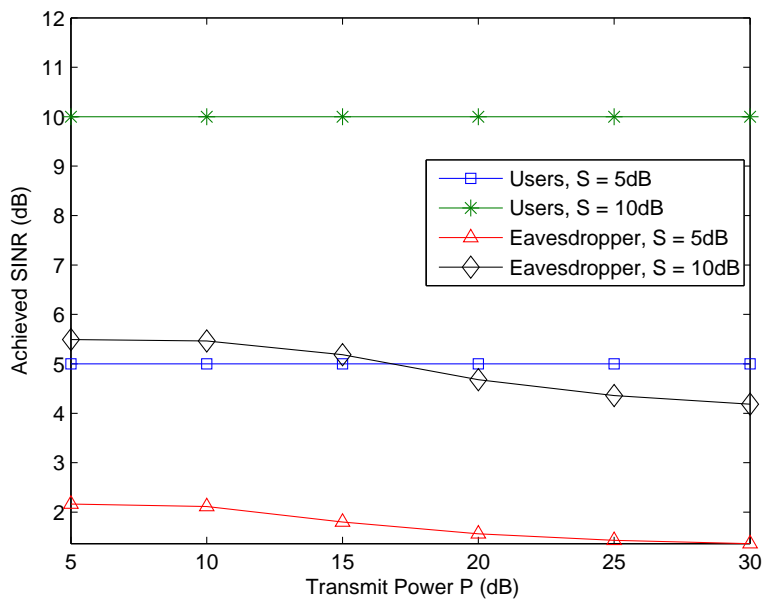

Fig. 5. SINR versus transmit power $P$ for $K=3$ users, $N_{t}=$ $4, N_{r}=2, N_{e}=4$ antennas.

formance is degraded. However, the degradation in the eavesdropper's SINR is not as severe as in the broadcast case since there is no multiuser interference to compound the effect of the artificial noise.

\section{CONCLUSION}

This paper has examined beamforming strategies combined with artificial noise for providing confidentiality at the physical layer in multiuser MIMO wiretap channels. For the MIMO broadcast channel with single-user detection at the eavesdropper, the zero-forcing beamformer design is shown to provide an acceptable level of performance in terms of relative SINR when compared to optimal joint transmit-receive beamforming algorithms. The use of artificial noise is meaningful even when the eavesdropper employs optimal ML joint detection for the information vector. For the MIMO multicast channel, the degradation in the eavesdropper's SINR as the transmit power increases is not as severe, but artificial noise is still seen to be effective.

\section{REFERENCES}

[1] A. D. Wyner, "The wire-tap channel," Bell Syst. Tech. J., vol. 54, no. 8, pp. 1355-1387, Oct. 1975.

[2] I. Csiszar and J. Korner, "Broadcast channels with confidential messages," IEEE Trans. Inf. Theory, vol. 24, no. 3, pp. 339-348, May 1978.

[3] Y. Liang and H. V. Poor, "Multiple-access channels with confidential messages," vol. 54, no. 3, pp. 976-1002, March 2008.

[4] A. Khisti, A. Tchamkerten, and G. W. Wornell, "Secure broadcasting over fading channels," IEEE Trans. Inf. Theory, vol. 54, no. 6, pp. 2453-2469, June 2008.

[5] Y. Liang, H. V. Poor, and S. Shamai, "Secure communication over fading channels," IEEE Trans. Inf. Theory, vol. 54, no. 6, pp. 2470-2492, June 2008.
[6] R. Liu, I. Maric, P. Spasojevic, and R. D. Yates, "Discrete memoryless interference and broadcast channels with confidential messages: secrecy rate regions," IEEE Trans. Inf. Theory, vol. 54, no. 6, pp. 2493-2507, June 2008.

[7] R. Liu and H. V. Poor, "Secrecy capacity region of a multipleantenna Gaussian broadcast channel with confidential messages," IEEE Trans. Inf. Theory, vol. 55, no. 3, pp. 1235-1249, March 2009.

[8] S. Goel and R. Negi, "Guaranteeing secrecy using artificial noise," IEEE Trans. Wireless Commun., vol. 7, no. 6, pp. 21802189, June 2008.

[9] A. L. Swindlehurst, "Fixed SINR solutions for the MIMO wiretap channel," Proc. of IEEE ICASSP, pp. 2437-2440, April 2009.

[10] Z. Pan, K. Wong, and T. Ng, "Generalized multiuser orthogonal space-division multiplexing," IEEE Trans. Wireless Commun., vol. 3, no. 6, pp. 1969-1973, Nov. 2004.

[11] Q. Spencer, A. L. Swindlehurst, and M. Haardt, "Zero-forcing methods for downlink spatial multiplexing in multiuser MIMO channels," IEEE Trans. Signal Process., vol. 52, pp. 462-471, Feb. 2004.

[12] J. Chang, L. Tassiulas, and F. Rashid-Farrokhi, "Joint transmitter receiver diversity for efficient space division multiaccess," IEEE Trans. Wireless Commun., vol. 1, no. 1, pp. 16-27, Jan. 2002.

[13] D. P. Palomar and M. A. Lagunas, "Joint transmit-receive space-time equalization in spatially correlated MIMO channels: a beamforming approach," IEEE J. Sel. Areas Commun.,, vol. 21, no. 5, pp. 730-743, June 2003.

[14] Q. Spencer and A. Swindlehurst, "A hybrid approach to spatial multiplexing in multi-user MIMO downlinks," EURASIP Journ. Wireless Commun. and Network., pp. 236-247, Dec. 2004.

[15] M. Codreanu, A. Tölli, M. Juntti, and M. Latva-aho, "Joint design of tx-rx beamformers in MIMO downlink channel," IEEE Trans. Signal Process., vol. 55, no. 9, pp. 4639-4655, Sep. 2007.

[16] N.D. Sidiropoulos, T.N. Davidson, and Z. Q. Luo, "Transmit beamforming for physical layer multicasting", IEEE Trans. Signal Process., vol. 54, no. 6, Part 1, pp. 2239-2251, June 2006.

[17] E. Karipidis, N.D. Sidiropoulos, Z. Q. Luo, "Quality of service and max-min-fair transmit beamforming to multiple co-channel multicast groups", IEEE Trans. Signal Process., vol. 56, no. 3, pp. 1268-1279, Mar. 2008.

[18] S. Shi, M. Schubert and H. Boche, "Physical layer multicasting with linear MIMO transceivers", in Proc. CISS, Princeton University, pp. 884 - 889, March 2008.

[19] C. B. Chae, D. Mazzarese, T. Inoue, and R. W. Heath, "Coordinated beamforming for the multiuser MIMO broadcast channel with limited feedforward," IEEE Trans. Signal Process., vol. 56, pp. 6044-6056, Dec. 2008.

[20] G. Zheng, K. K. Wong, and N. Tung-Sang, "Robust linear MIMO in the downlink : A worst-case optimization with ellipsoidal uncertainty regions," EURASIP J. Adv. Signal Process., pp. 1-15, 2008.

[21] A. Mukherjee and A. L. Swindlehurst, "Robust Beamforming for Secrecy in MIMO Wiretap Channels with Imperfect CSI," submitted to IEEE Trans. Signal Process., July 2009.

[22] A. Mukherjee and A. L. Swindlehurst, "User selection in multiuser MIMO Systems with secrecy considerations," submitted to ASILOMAR Conf. on Signals, Systems, and Computers 2009, Pacific Grove, CA.

[23] M. Grant, S. Boyd, and Y. Ye, "CVX: Matlab software for disciplined convex programming," 2005. 